\title{
Time Period of the Vibration of the Circular Plate with Circular Variation Both in Thickness and Density
}

\author{
Reeta Bhardwaj, ${ }^{1}$ Amit Sharma $\mathbb{D}^{1},{ }^{1}$ Sudeshna Ghosh, ${ }^{1}$ Naveen Mani $\oplus{ }^{2}$ \\ and Kamal Kumar $\mathbb{D}^{1}$ \\ ${ }^{1}$ Department of Mathematics, Amity School of Applied Sciences, Amity University Haryana, Gurugram, Haryana, India \\ ${ }^{2}$ Department of Mathematics, Chandigarh University, Mohali, India \\ Correspondence should be addressed to Amit Sharma; dba.amitsharma@gmail.com and Kamal Kumar; kamalkumarrajput92@ \\ gmail.com
}

Received 16 July 2021; Revised 26 September 2021; Accepted 17 November 2021; Published 13 December 2021

Academic Editor: Alessandro Lo Schiavo

Copyright (c) 2021 Reeta Bhardwaj et al. This is an open access article distributed under the Creative Commons Attribution License, which permits unrestricted use, distribution, and reproduction in any medium, provided the original work is properly cited.

\begin{abstract}
An analysis was carried out to investigate the time period of the thermally induced vibration of clamped and simply supported circular plates with circular variation both in thickness and density. Prior to this study, the variations considered were either linear, quadratic, parabolic, or exponential in nature. To study thermal effect, one-dimensional linear temperature variation on the plates is taken into consideration. Rayleigh-Ritz method is applied to compute the time period of the first three modes of vibration for both plates by varying tapering parameter, thermal gradient, and density. Convergence study of frequency modes for both plates conducted suggests that the convergence rate in case of circular variation is faster than the other studies done. A comparison of time period with the available published results is done. The comparison done concludes that time period obtained in the present study by varying thermal gradient and tapering parameter is found to be less than the other studies done for the same set of parameters. This study helped to establish the fact that, by using circular variation in plate parameters, we can get less time period of frequency modes in comparison to other variations considered till date.
\end{abstract}

\section{Introduction}

Vibration, sometimes, is described as a kind of waste energy. In modern days, vibrational study is involved in a wide range of industrial applications and research. Study of natural vibration of nonuniform plates is very essential because vibration is used in many engineering and science applications. Different shapes of plates are the choices of many engineering applications, depending on the requirement of the systems. Also, different shapes of plates with different types of variations in plate parameters are used according to the choices of researchers in order to make good structural designs. The study of the vibration of plates such as circular, elliptical, square, rectangular, and skew plates with linear and nonlinear variations in thickness, density, and temperature has been carried out by many researchers and is well documented in the literature. To the best of our knowledge, vibration of circular plates with circular variations in both thickness and density has not been considered yet.

The problem of bending and vibration of a simply supported rectangular plate with linearly varying thickness is discussed in [1]. The differential quadrature method was implemented to study the natural vibrations of clamped, simply supported, and free circular plates (nonhomogeneous and isotropic) of nonlinear thickness variation and computed first three frequencies [2]. An exact solution of inhomogeneous circular plates has been discussed by using the novel analytical method, and the effect of Poisson's ratio, rotatory inertial, and shear deformation is examined [3]. The Rayleigh-Ritz method along with characteristic orthogonal polynomials has been applied to study the fundamental mode of vibrations of circular or elliptic plates [4]. An analysis of the vibration of circular annular disks on the clamped, free, and flexible boundary at the inner and outer edges has been studied, and the studies focused on frequency 
parameters for various plate parameters [5]. The Ritz method along with Chebyshev polynomials has been used to study the effect of different material compositions, Poisson's ratio, and the plate geometry on the frequencies and mode shapes of FGM plates [6]. The vibration of FG isotropic circular plates with exponential thickness has been studied using the displacement method on two edge conditions [7]. The effects of material heterogeneity and multifield coupling on the static behavior of the FG circular plate made of MEE materials under tension and bending have been discussed [8]. Levy approach and quintic spline method have been used to examine the effect of damping, elastic foundation, and taperness on damped vibrations of the homogeneous rectangular plate of linearly varying thickness resting on an elastic foundation [9]. Vibrations of FG annular plates on the ring support have been analyzed and presented numerically on the basis of classical plate theory [10]. The effect of exponential Young's modulus and density on the asymmetric vibrations of nonhomogeneous, clamped, simply supported, and free circular plates with parabolic thickness has been studied using the Ritz method, and the first three natural frequencies have been presented [11]. The vibration of the nonuniform skew plate with both circular variation in density and Poisson's ratio and the natural vibration of the nonuniform skew plate with both circular variation in thickness and Poisson's ratio have been analyzed using the Rayleigh-Ritz method under the temperature field, and frequency modes comprising the effect of various plate parameters have been computed $[12,13]$. The effect of circular variation in Poisson's ratio on frequencies of the nonuniform rectangle plate and effect of circular variation in density on frequencies of the nonuniform square plate have been presented using the Rayleigh-Ritz method under the temperature field $[14,15]$. Rayleigh-Ritz method is used to analyze the vibration of the circular plate with linearly varying thickness and temperature; and deflection, time period, and logarithmic decrement have been computed for different values of plate parameters [16]. Generalized differential quadrature method is applied to study free thermal vibrations of FGM; clamped and simply supported circular plates and first three modes of vibration have been computed [17].

In this study, time period of frequency modes (first three) comprises the effect of various plate parameters (circular variation in both density and thickness and linear variation in thermal gradient) for clamped and simply supported circular plates, and the result is presented in the form of tables and graphs. As far the knowledge of the authors, prior to this work, the effect of circular variation in plate parameters on the circular plate was not examined. In order to authenticate the findings of the present study, a comparative study of time period is conducted with the available published results for the clamped circular plate.

This paper has been organized in the following manner. First, mathematical formulation of the problem has been discussed, and then the numerical results have been reported. In the end, the main conclusions of the studies have been discussed.

\section{Analysis}

Consider a circular plate of radius $r_{1}$ having circular thickness $l(r)$, circular density $\rho(r)$, and Poisson's ratio $v$ referred to cylindrical polar coordinates $(r, \theta, z)$ (refer to Figure 1)

The maximum strain energy $V_{s}$ and kinetic energy $T_{s}$ of the plate are given by the expression derived in [18]:

$$
V_{s}=\frac{1}{2} \int_{0}^{2 \pi} \int_{0}^{r_{1}} D\left[\begin{array}{c}
\left\{\frac{\partial^{2} \phi}{\partial r^{2}}+\frac{1}{r} \frac{\partial \phi}{\partial r}+\frac{1}{r^{2}} \frac{\partial^{2} \phi}{\partial \theta^{2}}\right\}^{2} \\
-2(1-v)\left\{\frac{\partial^{2} \phi}{\partial r^{2}}\left(\frac{1}{r} \frac{\partial \phi}{\partial r}+\frac{1}{r^{2}} \frac{\partial^{2} \phi}{\partial \theta^{2}}\right)\right. \\
\left.-\left(\frac{\partial}{\partial r}\left(\frac{1}{r} \frac{\partial \phi}{\partial \theta}\right)\right)^{2}\right\}
\end{array}\right] r \mathrm{~d} r \mathrm{~d} \theta,
$$

where $D=E l^{3} / 12\left(1-v^{2}\right)$ is the flexural rigidity, $E$ is Young's modulus, and $\phi$ is known as the transverse deflection.

$$
T_{s}=\frac{1}{2} \omega^{2} \int_{0}^{2 \pi} \int_{0}^{r_{1}} \rho l \phi^{2} r \mathrm{~d} r \mathrm{~d} \theta
$$

where $\omega$ is the circular frequency.

Applying the Rayleigh-Ritz method, the variable $J$ is represented in the following manner:

$$
J=\delta\left(V_{s}-T_{s}\right)=0 .
$$

Substituting equations (1) and (2) in equation (3), we get

$$
J(\phi)=\frac{1}{2} \int_{0}^{2 \pi} \int_{0}^{r_{1}} D\left[\begin{array}{c}
\left\{\frac{\partial^{2} \phi}{\partial r^{2}}+\frac{1}{r} \frac{\partial \phi}{\partial r}+\frac{1}{r^{2}} \frac{\partial^{2} \phi}{\partial \theta^{2}}\right\}^{2} \\
-2(1-\nu)\left\{\frac{\partial^{2} \phi}{\partial r^{2}}\left(\frac{1}{r} \frac{\partial \phi}{\partial r}+\frac{1}{r^{2}} \frac{\partial^{2} \phi}{\partial \theta^{2}}\right)\right. \\
\left.-\left(\frac{\partial}{\partial r}\left(\frac{1}{r} \frac{\partial \phi}{\partial \theta}\right)\right)^{2}\right\}
\end{array}\right] r \mathrm{~d} r \mathrm{~d} \theta
$$

Introducing nondimensional variable $\Phi=\phi / r_{1}$ and $R=$ $r / r_{1}$ along with circular variation in both density $\rho$ and thickness $l$, we get

$$
\begin{aligned}
& \rho=\rho_{0}\left\{1-\varphi\left(1-\sqrt{1-R^{2}}\right)\right\}, \\
& l=l_{0}\left\{1-\oplus\left(1-\sqrt{1-R^{2}}\right)\right\},
\end{aligned}
$$

where $\rho_{0}$ and $l_{0}$ are nonhomogeneity and tapering parameters of the plate, respectively. 


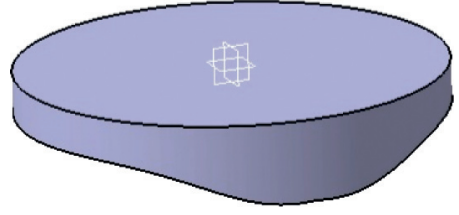

FIgURE 1: Circular plate with circular variation in thickness.

In [16], the temperature was assumed to be linear in the radial direction. In this study, we also assumed the same. Under this assumption, the temperature of the plate takes the form

$$
\eta=\eta_{0}(1-R)
$$

where $\eta$ and $\eta_{0}$ are the temperature on the plate and at the center of the plate, respectively. The temperature-dependent modulus of elasticity is taken as in [19] and can be expressed in the following manner:

$$
E=E_{0}(1-\varrho \eta)
$$

where $\varrho$ is the slope of variation and $E_{0}$ is Young's modulus at $\eta=0$. Substituting equation (6) in equation (7),

$$
E=E_{0}\{1-\kappa(1-R)\},
$$

where $\kappa=\varrho \eta_{0}$ is the thermal gradient.

Using nondimensional variables $\Phi$ and $R$ and substituting equations (5) and (8) in equation (4), we get

$$
\begin{aligned}
J(\Phi)= & \frac{D_{0}}{2} \int_{0}^{2 \pi} \int_{0}^{1}\left[\{1-\kappa(1-R)\}\left\{1-\Phi\left(1-\sqrt{1-R^{2}}\right)\right\}^{3}\right] \\
& {\left[\frac{\partial^{2} \Phi}{\partial R^{2}}+\frac{1}{R} \frac{\partial \Phi}{\partial R}+\frac{1}{R^{2}} \frac{\partial^{2} \Phi}{\partial \theta^{2}}\right\}^{2} } \\
-2(1-\nu)\left\{\frac{\partial^{2} \Phi}{\partial R^{2}}\left(\frac{1}{R} \frac{\partial \Phi}{\partial R}+\frac{1}{R^{2}} \frac{\partial^{2} \Phi}{\partial \theta^{2}}\right)\right. & \left.-\left(\frac{\partial}{\partial R}\left(\frac{1}{R} \frac{\partial \Phi}{\partial \theta}\right)\right)^{2}\right\} \\
& {\left[\lambda _ { 0 } ^ { 2 \pi } \int _ { 0 } ^ { 1 } \left[\left\{1-\Phi\left(1-\sqrt{1-R^{2}}\right)\right\}\right.\right.}
\end{aligned}
$$

where $D_{0}=E_{0} l_{0}^{3} / 12\left(1-v^{2}\right)$ and $\lambda^{2}=\rho_{0} a^{4} \omega^{2} l_{0} / D_{0}$.

Now, assuming the mode shape function as in [20], we have

$$
\Phi(R)=\Phi_{1}(R) \cos n \theta .
$$

As the functional in equation (9) contains the negative power of $R$, take $\Phi_{1}(R)=R \overline{\Phi_{1}}(R)$ as in [16], and using this relation and substituting equation (10) in equation (9), we get

$$
\begin{aligned}
& J\left(\overline{\Phi_{1}}\right)= \frac{D_{0}}{2} \int_{0}^{1}\left[\{1-\kappa(1-R)\}\left\{1-\omega\left(1-\sqrt{1-R^{2}}\right)\right\}^{3}\right] \\
& \times\left[3 \frac{\mathrm{d} \overline{\Phi_{1}}}{\mathrm{~d} R}+R \frac{\mathrm{d}^{2} \overline{\Phi_{1}}}{\mathrm{~d} R^{2}}\right\}^{2} \\
&\left.-2(1-\nu)\left\{\frac{\mathrm{d} \overline{\Phi_{1}}}{\mathrm{~d} R}\left(\frac{\mathrm{d} \overline{\Phi_{1}}}{\mathrm{~d} R}+R \frac{\mathrm{d}^{2} \overline{\Phi_{1}}}{\mathrm{~d} R^{2}}\right)\right\}\right] R \mathrm{~d} R \\
&-\lambda^{2} \int_{0}^{1}\left[\left\{1-\Phi\left(1-\sqrt{1-R^{2}}\right)\right\}\right. \\
&\left.\left\{1-\varphi\left(1-\sqrt{1-R^{2}}\right)\right\} \bar{\Phi}_{1}^{2}\right] R^{3} \mathrm{~d} R .
\end{aligned}
$$

We choose deflection function, $\overline{\Phi_{1}}$, in the radial direction which satisfies the geometric boundary condition as

$$
\overline{\Phi_{1}}=(1-R)^{p} \sum_{i=0}^{N} C_{i}(1-R)^{i},
$$

where $p$ can be 0,1 , and 2 in accordance to free, simply supported, and clamped boundary conditions, respectively. $C_{i}, i=0,1,2, \ldots, N$, are arbitrary constants.

In order to minimize equation (11), we require the following condition:

$$
\frac{\partial J\left(\overline{\Phi_{1}}\right)}{\partial C_{i}}=0, \quad i=0,1,2, \ldots N
$$

From equation (13), we have a system of homogeneous equations in $C_{i}$ whose nonzero solution gives the frequency equation as

$$
\left|P-\lambda^{2} Q\right|=0,
$$

where $P=\left[p_{i j}\right]_{N+1}$ and $Q=\left[q_{i j}\right]_{N+1}$ are square matrices and $i, j=0,1,2, \ldots, N$.

The time period $K$ is calculated as

$$
K=\frac{2 \pi}{\lambda},
$$

where $\lambda$ is the frequency obtained from equation (14).

\section{Numerical Results}

In this section, we will report the results obtained from numerical simulations.

3.1. Time Period Analysis. The time period, $K$, comprising the effect of circular variation in both thickness and density is calculated for clamped and simply supported circular plates under the linear temperature variation effect, and results are presented with the help of tables. The value of Poisson's ratio $v=0.345$ throughout the calculation.

Table 1 shows the time period $K$ for clamped and simply supported circular plates corresponding to tapering parameter $\omega$, for the variable value of thermal gradient $\kappa$ and 
TABLE 1: Time period $K$ of clamped and simply supported circular plates vs. tapering parameter $₫$.

\begin{tabular}{|c|c|c|c|c|c|c|c|}
\hline & \multirow{2}{*}{ 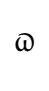 } & \multicolumn{3}{|c|}{$\kappa=0.0, \varphi=0.2$} & \multicolumn{3}{|c|}{$\kappa=0.4, \varphi=0.6$} \\
\hline & & $K_{1}$ & $K_{2}$ & $K_{3}$ & $K_{1}$ & $K_{2}$ & $K_{3}$ \\
\hline \multirow{5}{*}{ Clamped } & 0.0 & 0.03983 & 0.09867 & 0.29113 & 0.04583 & 0.10351 & 0.30845 \\
\hline & 0.2 & 0.04040 & 0.10616 & 0.31457 & 0.04703 & 0.11164 & 0.33260 \\
\hline & 0.4 & 0.04080 & 0.11416 & 0.34300 & 0.04797 & 0.12081 & 0.36194 \\
\hline & 0.6 & 0.04103 & 0.12223 & 0.37753 & 0.04863 & 0.13056 & 0.39782 \\
\hline & 0.8 & 0.04111 & 0.12978 & 0.41808 & 0.04902 & 0.14017 & 0.44080 \\
\hline \multirow{5}{*}{ Simply supported } & 0.0 & 0.04107 & 0.11052 & 0.44102 & 0.04024 & 0.12179 & 0.46317 \\
\hline & 0.2 & 0.04579 & 0.11531 & 0.46445 & 0.04567 & 0.12609 & 0.48783 \\
\hline & 0.4 & 0.05091 & 0.12215 & 0.49088 & 0.05204 & 0.13182 & 0.51535 \\
\hline & 0.6 & 0.05595 & 0.13237 & 0.52169 & 0.05908 & 0.14033 & 0.54692 \\
\hline & 0.8 & 0.06025 & 0.14754 & 0.56030 & 0.06605 & 0.15370 & 0.58519 \\
\hline
\end{tabular}

nonhomogeneity $\varphi$, i.e., $\kappa=0.0, \varphi=0.2$ and $\kappa=0.4, \varphi=0.6$. It can be easily seen that time period $K$ is increasing for the increasing value of tapering parameter $₫$ for all the aforementioned values of thermal gradient $\kappa$ and nonhomogeneity $\varphi$. The time period $K$ is also increasing when thermal gradient $\kappa$ as well as nonhomogeneity $\varphi$ increases from 0.0 to 0.4 and 0.2 to 0.6 , respectively. The time period $K$ of the simply supported circular plate is higher when compared with the time period $K$ of the clamped circular plate. Also, the rate of increment in time period $K$ for the simply supported circular plate is much higher in comparison to the rate of increments in time period $K$ for the clamped circular plate.

Time period, $K$, for clamped and simply supported circular plates corresponding to thermal gradient $\kappa$, for the variable value of tapering parameter $\omega$ and nonhomogeneity $\varphi$, i.e., $\Phi=0.0, \varphi=0.2$ and $\Phi=0.4, \varphi=0.6$, is presented in Table 2. One can conclude that increasing value of thermal gradient $\kappa$ causes the increase in time period $K$ for all the aforementioned values of tapering parameter $\omega$ and nonhomogeneity $\varphi$. The time period $K$ is also increasing when thermal gradient $\kappa$ as well as nonhomogeneity $\varphi$ increases from 0.0 to 0.4 and 0.2 to 0.6 , respectively. The behavior of time period $K$ of the simply supported circular plate is the same as the behavior of time period $K$ reported in Table 1 , but for the clamped plate, the time period $K$ of the first mode is slightly less for $\omega=0.0, \varphi=0.2$ than the time period $K$ of the first mode on $\Phi=0.4, \varphi=0.6$ at $\kappa=0.0$. Here, the rate of increment in time period $K$ for the clamped circular plate is much lesser in comparison to the rate of increments in time period $K$ for the simply supported circular plate.

Table 3 displays the time period $K$ for clamped and simply supported circular plates corresponding to nonhomogeneity $\varphi$, for the variable value of thermal gradient $\kappa$ and tapering parameter $\bowtie$, i.e., $\kappa=0.0, \Phi=0.2$ and $\kappa=0.4, \Phi=0.6$. Table 3 concludes that increasing value of nonhomogeneity $\varphi$ causes the decrease in time period $K$ for the aforementioned variable value of thermal gradient $\kappa$ and tapering parameter $\omega$, but the time period $K$ for both clamped and simply supported circular plates increases when the value of thermal gradient $\kappa$ and tapering parameter $₫$ varies from 0.0 to 0.2 and 0.2 to 0.6 , respectively. The rate of decrement in time period $K$ for the clamped circular plate is much lesser when compared to the rate of decrement in time period $K$ for the simply supported circular plate.
In view to understand the obtained results as discussed in this section, a graphical illustration of results is given (refer to Figures 2-7).

3.2. Convergence Studies. In this section, we will focus on the convergence studies done for clamped and simply supported circular plates computed for plate parameters in the range specified. The results are shown in Figure 8 for $\varphi=\emptyset=\kappa=$ 0.0 and $\nu=0.345$.

The convergence study is done for two modes $\lambda_{1}$ and $\lambda_{2}$ of vibrations for both clamped and simply supported circular plates. It was observed that, for approximately $N=7$ onwards, the value of the modes was constant up to five decimal places. The aforementioned results show that the modes converge.

3.3. Results' Comparison. In order to validate the findings of the present study, a graphical comparison of time period $K$ of the clamped circular plate is given with the available published results corresponding to tapering parameter $\varpi$ and nonhomogeneity $\varphi$.

Figure 9 presents the comparison of time period $K$ of the clamped circular plate with the time period $K$ obtained in [16] corresponding to tapering parameter $₫$ for fixed values of thermal gradient $\kappa=0.0$ and nonhomogeneity $\varphi=0.0$. From Figure 9, one can easily conclude that time period $K$ as well as variation in time period (rate of increment) $K$ of the present study is less when compared with the time period $K$ obtained in [16] for both fixed values of thermal gradient $\kappa$ and nonhomogeneity $\varphi$.

A comparison of time period $K$ of the clamped circular plate with the time period $K$ obtained in [16] corresponding to nonhomogeneity $\varphi$ for fixed values of thermal gradient $\kappa=0.0$ and tapering $\omega=0.0$ is presented in Figure 10. Here, the time period $K$ of the present study is higher than the time period $K$ obtained in [16] for fixed values of thermal gradient $\kappa$ and tapering parameter $\omega$, but the variation in time period $K$ (rate of decrement) of the present study is much lesser when compared with the variation of time period $K$ obtained in $[16]$. 
TABLE 2: Time period $K$ of clamped and simply supported circular plates vs. thermal gradient $\kappa$.

\begin{tabular}{|c|c|c|c|c|c|c|c|}
\hline & \multirow{2}{*}{$\kappa$} & \multicolumn{3}{|c|}{$\Phi=0.0, \varphi=0.2$} & \multicolumn{3}{|c|}{$\omega=0.4, \varphi=0.6$} \\
\hline & & $K_{1}$ & $K_{2}$ & $K_{3}$ & $K_{1}$ & $K_{2}$ & $K_{3}$ \\
\hline \multirow{5}{*}{ Clamped } & 0.0 & 0.03983 & 0.09867 & 0.29113 & 0.03972 & 0.10937 & 0.33194 \\
\hline & 0.2 & 0.04313 & 0.10225 & 0.30311 & 0.04327 & 0.11458 & 0.34561 \\
\hline & 0.4 & 0.04736 & 0.10799 & 0.31749 & 0.04797 & 0.12081 & 0.36194 \\
\hline & 0.6 & 0.05307 & 0.11487 & 0.33537 & 0.05458 & 0.12869 & 0.38221 \\
\hline & 0.8 & 0.06118 & 0.12576 & 0.35905 & 0.06481 & 0.14000 & 0.40900 \\
\hline \multirow{5}{*}{ Simply supported } & 0.0 & 0.04107 & 0.11052 & 0.44102 & 0.04793 & 0.11644 & 0.47030 \\
\hline & 0.2 & 0.04215 & 0.11755 & 0.46021 & 0.04988 & 0.12306 & 0.49100 \\
\hline & 0.4 & 0.04330 & 0.12669 & 0.48280 & 0.05204 & 0.13182 & 0.51535 \\
\hline & 0.6 & 0.04452 & 0.13920 & 0.51045 & 0.05442 & 0.14416 & 0.54540 \\
\hline & 0.8 & 0.04582 & 0.15771 & 0.54654 & 0.05706 & 0.16322 & 0.58340 \\
\hline
\end{tabular}

TABLE 3: Time period $K$ of clamped and simply supported circular plates vs. nonhomogeneity $\varphi$.

\begin{tabular}{|c|c|c|c|c|c|c|c|}
\hline & \multirow{2}{*}{$\varphi$} & \multicolumn{3}{|c|}{$\kappa=0.0, \Phi=0.2$} & \multicolumn{3}{|c|}{$\kappa=0.4, \Phi=0.6$} \\
\hline & & $K_{1}$ & $K_{2}$ & $K_{3}$ & $K_{1}$ & $K_{2}$ & $K_{3}$ \\
\hline \multirow{5}{*}{ Clamped } & 0.0 & 0.04091 & 0.10830 & 0.31934 & 0.05053 & 0.13873 & 0.41761 \\
\hline & 0.2 & 0.04040 & 0.10616 & 0.31457 & 0.04993 & 0.13612 & 0.41111 \\
\hline & 0.4 & 0.03986 & 0.10394 & 0.30972 & 0.04930 & 0.13340 & 0.40451 \\
\hline & 0.6 & 0.03927 & 0.10162 & 0.30482 & 0.04863 & 0.13056 & 0.39782 \\
\hline & 0.8 & 0.03865 & 0.09920 & 0.29986 & 0.04791 & 0.12760 & 0.39103 \\
\hline \multirow{5}{*}{ Simply supported } & 0.0 & 0.04709 & 0.11764 & 0.47388 & 0.06427 & 0.15046 & 0.58189 \\
\hline & 0.2 & 0.04579 & 0.11531 & 0.46445 & 0.06265 & 0.14722 & 0.57048 \\
\hline & 0.4 & 0.04440 & 0.11288 & 0.45487 & 0.06095 & 0.14385 & 0.55880 \\
\hline & 0.6 & 0.04288 & 0.11033 & 0.44507 & 0.05908 & 0.14033 & 0.54692 \\
\hline & 0.8 & 0.04120 & 0.10764 & 0.43508 & 0.05703 & 0.13663 & 0.53483 \\
\hline
\end{tabular}
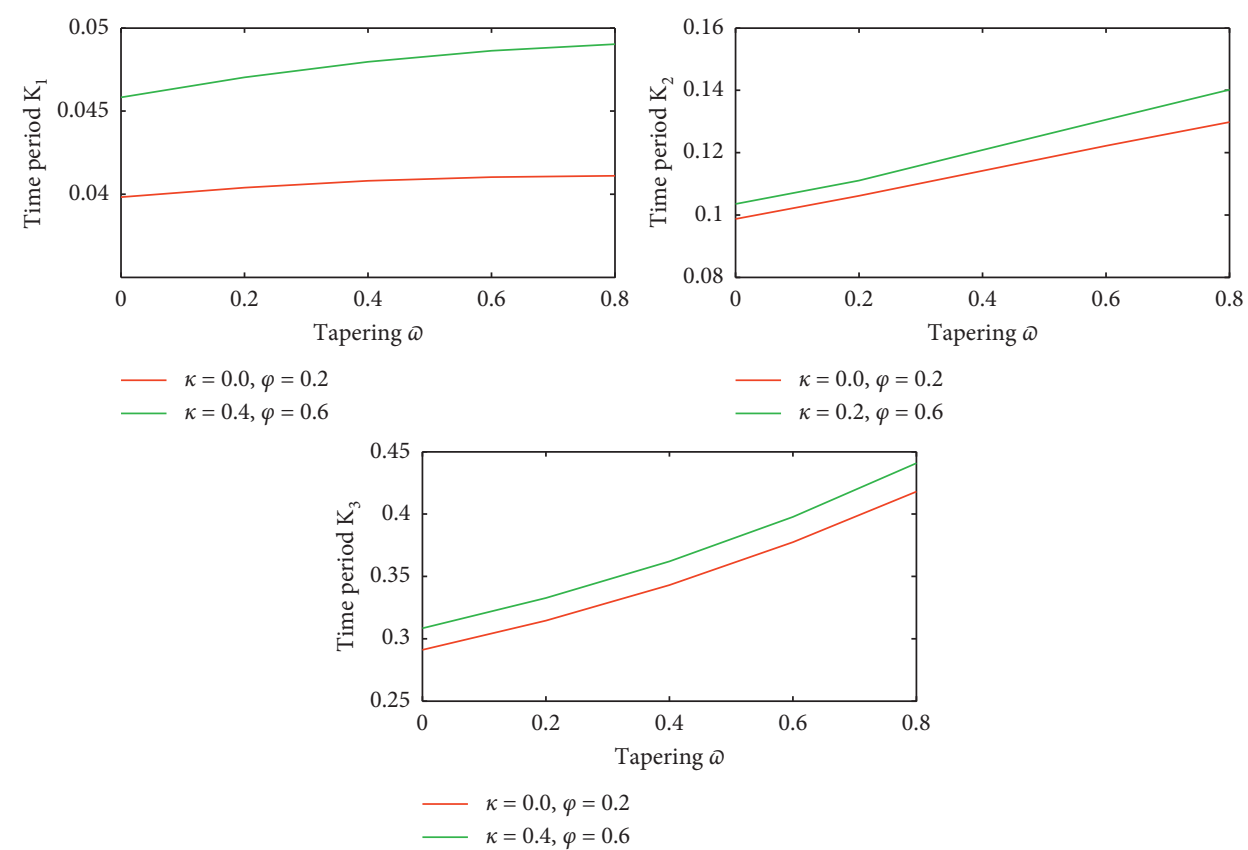

Figure 2: Time period $K$ of the clamped circular plate vs. tapering parameter $\varpi$. 

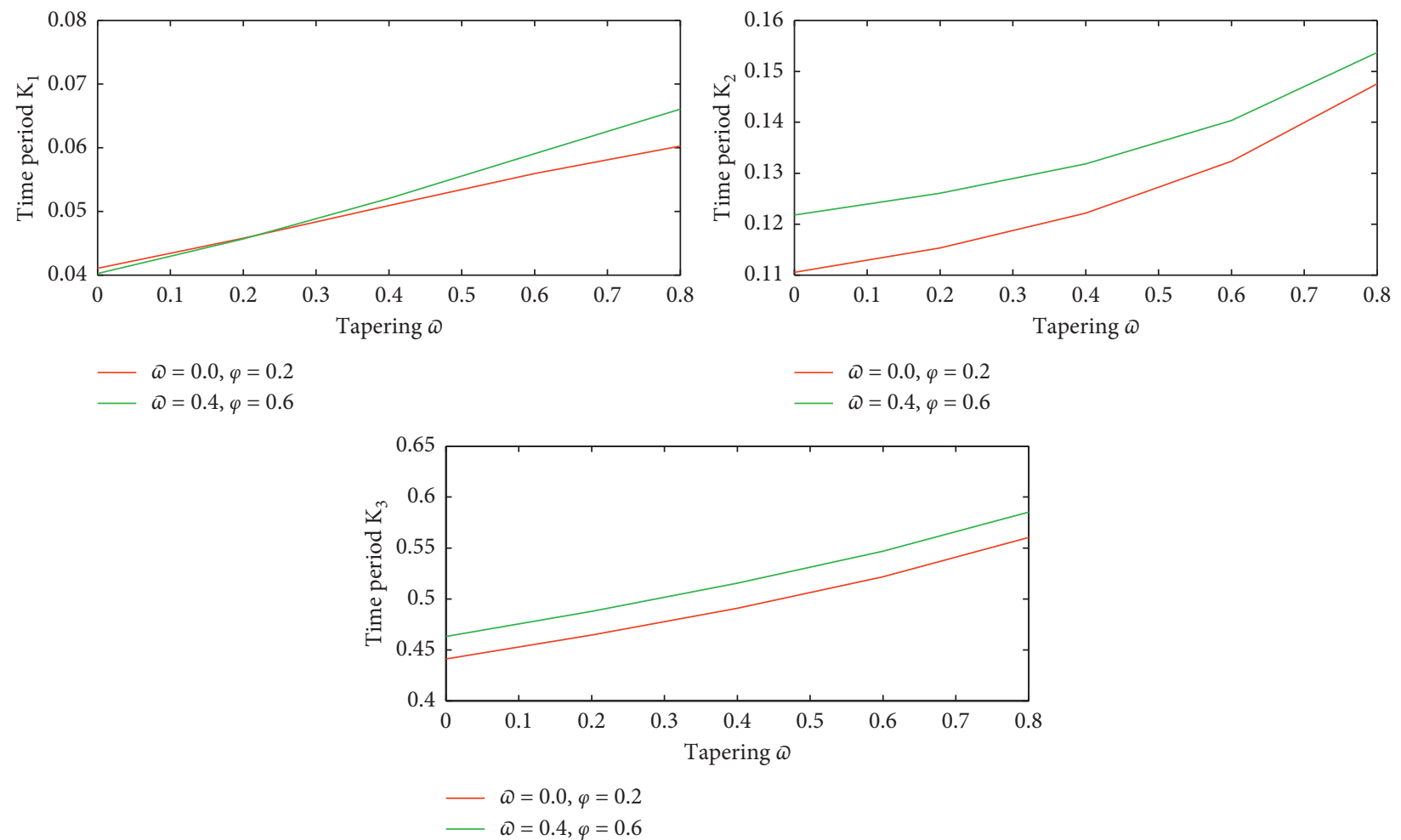

Figure 3: Time period $K$ of the simply supported circular plate vs. tapering parameter $\varpi$.
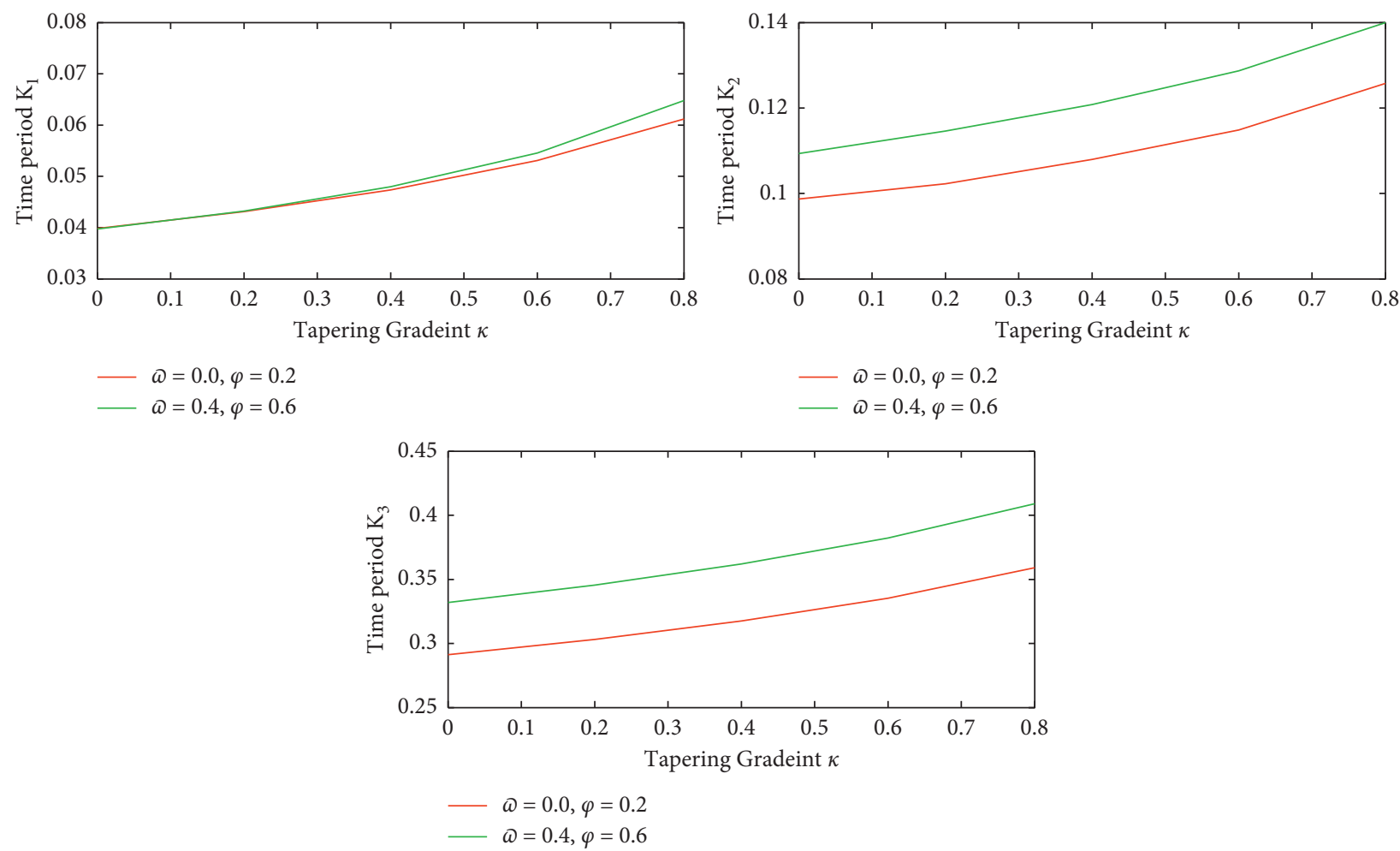

Figure 4: Time period $K$ of the clamped circular plate vs. thermal gradient $\kappa$. 

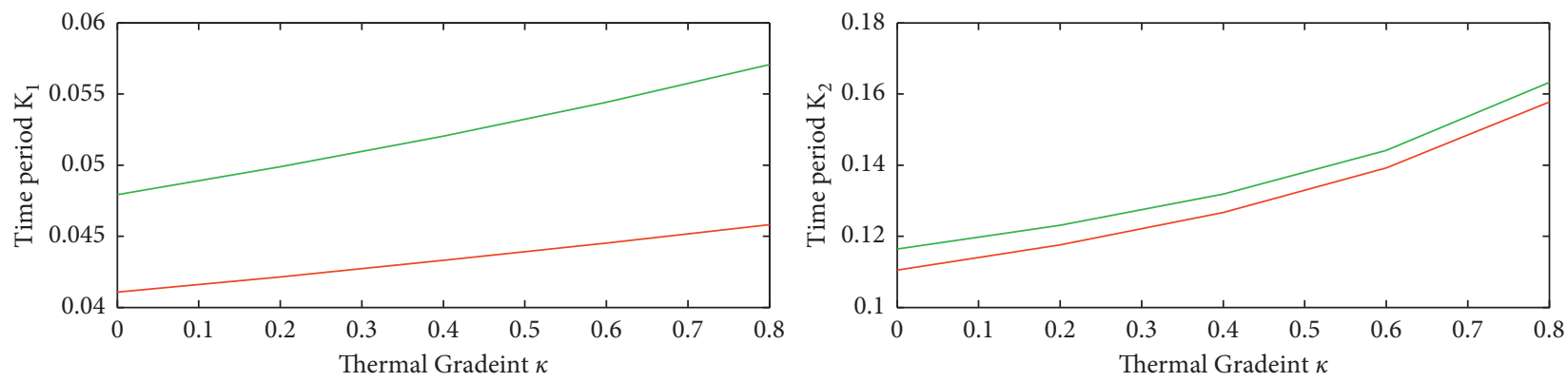

$\begin{aligned}-\omega & =0.0, \varphi=0.2 \\ \omega & =0.4, \varphi=0.6\end{aligned}$

$\begin{aligned}-\omega & =0.0, \varphi=0.2 \\ \omega & =0.4, \varphi=0.6\end{aligned}$

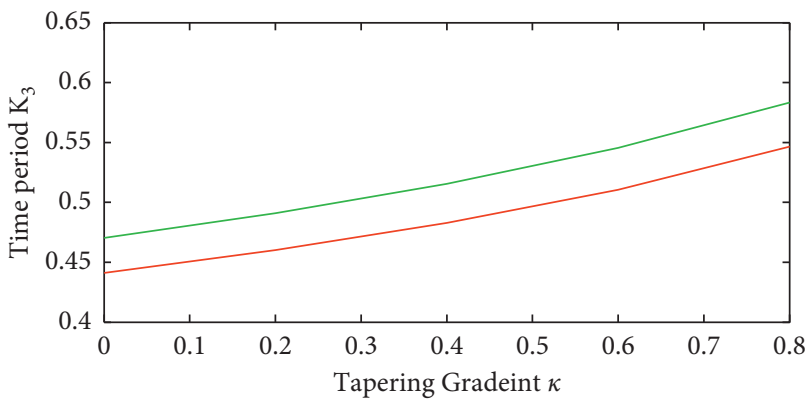

$-\omega=0.0, \varphi=0.2$

$\longrightarrow \omega=0.4, \varphi=0.6$

FIgURE 5: Time period $K$ of the simply supported circular plate vs. thermal gradient $\kappa$.
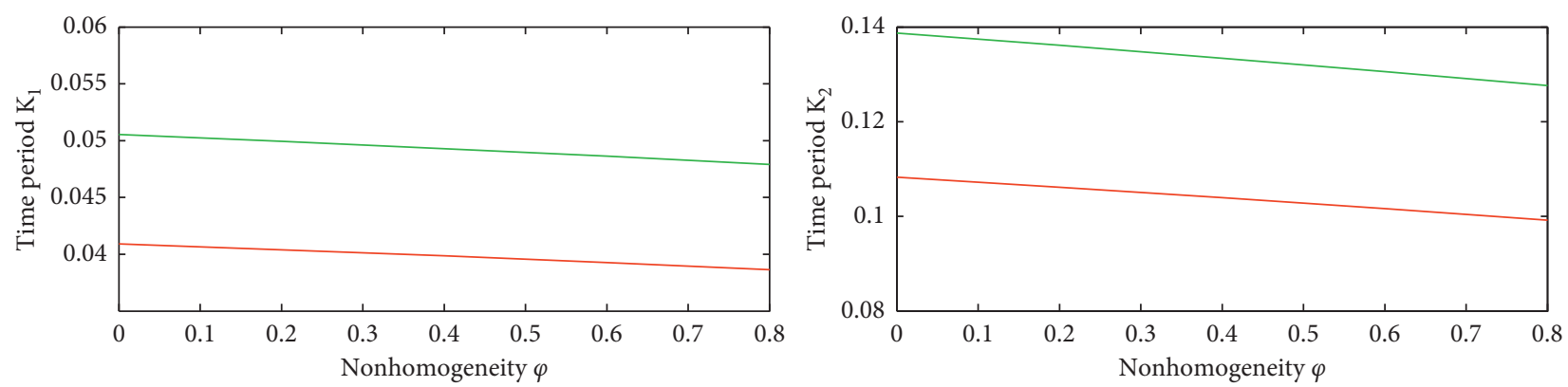

- $\kappa=0.0, \omega=0.2$

- $\kappa=0.0, \omega=0.2$

$\varkappa=0.4, \omega=0.6$

$\kappa=0.4, \omega=0.6$

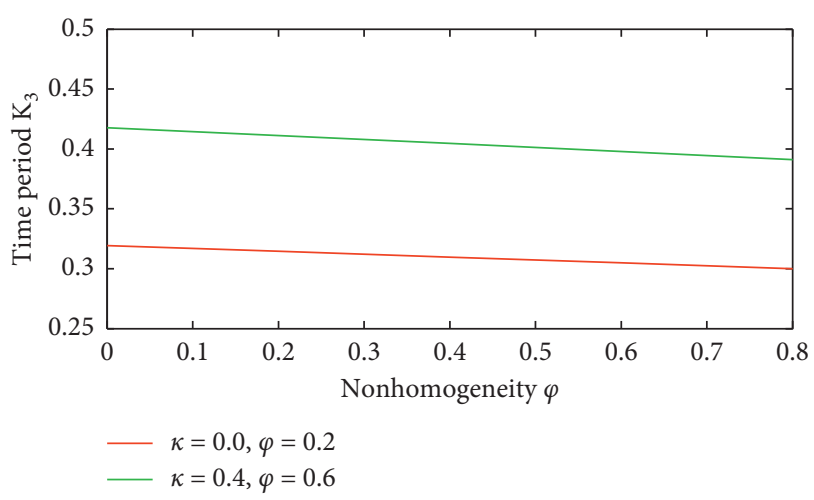

FIGURE 6: Time period $K$ of the clamped circular plate vs. nonhomogeneity $\varphi$. 

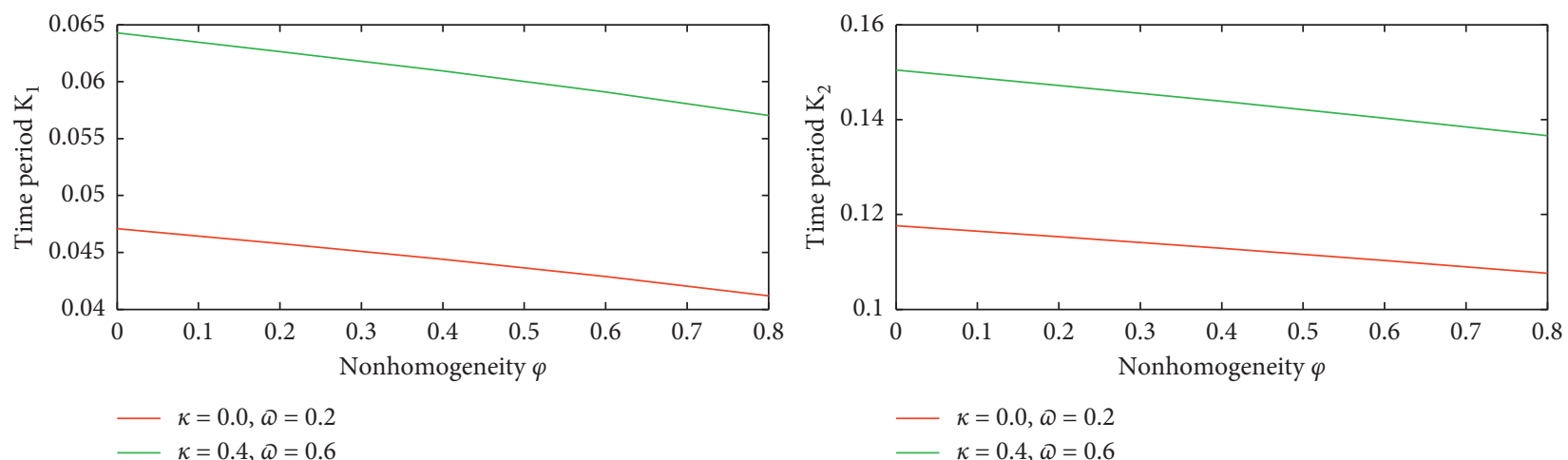

- $\kappa=0.0, \omega=0.2$

$\kappa=0.4, \omega=0.6$

$\leftarrow \kappa=0.4, \omega=0.6$

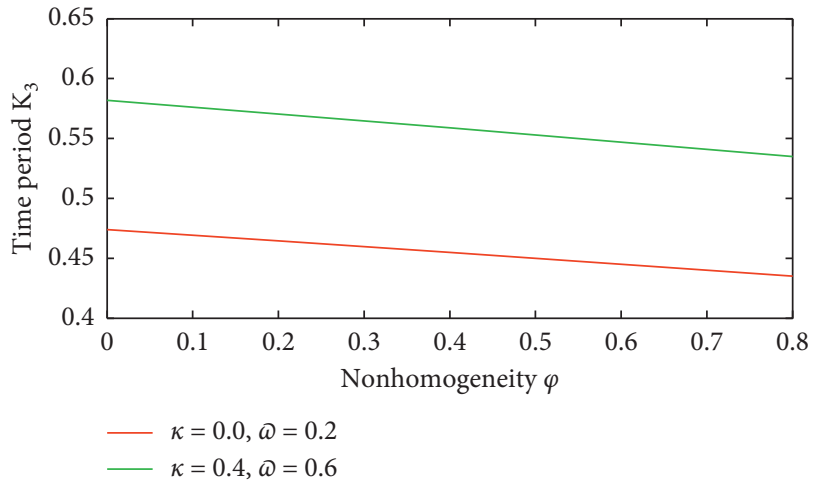

FIgURE 7: Time period $K$ of the simply supported circular plate vs. nonhomogeneity $\varphi$.
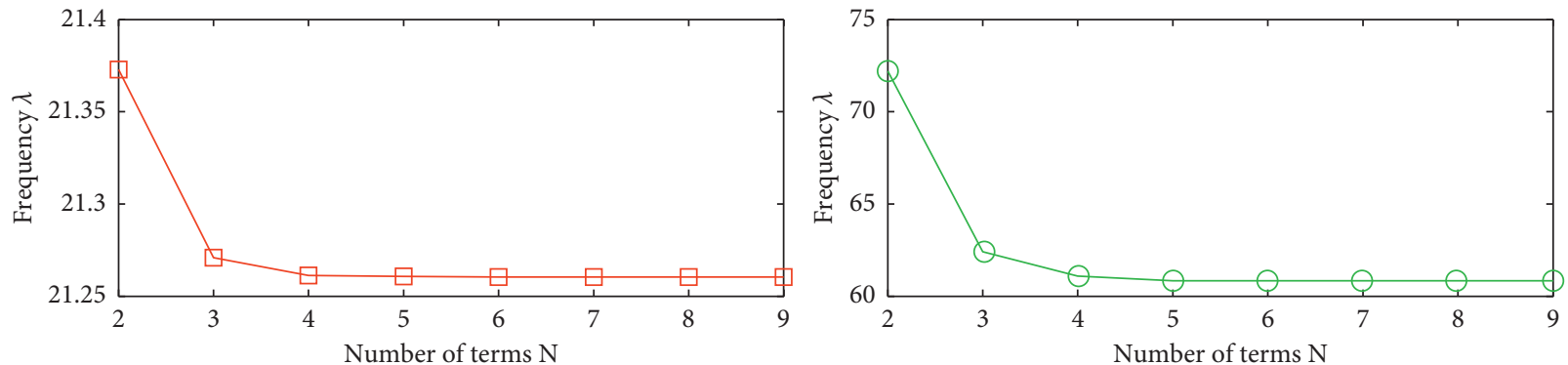

Clamped circular plate

$\square \lambda_{1}$

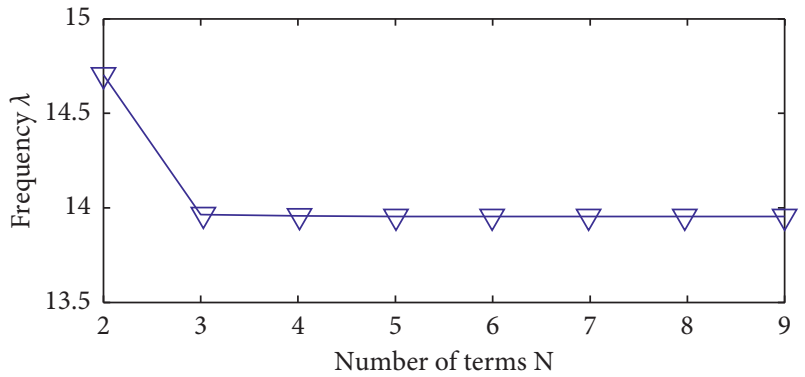

Clamped circular plate

$\multimap \lambda_{2}$

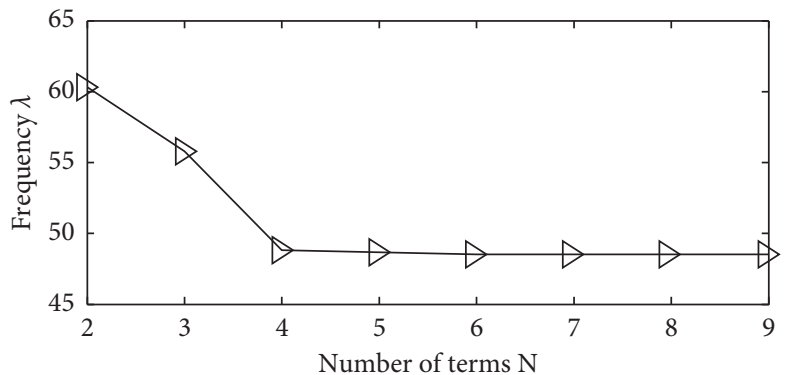

Simply supported circular plate

Simply supported circular plate

$\nabla \lambda_{1}$

$\rightarrow \lambda_{2}$

FIGURE 8: Convergence of frequency modes of clamped and simply supported circular plates. 


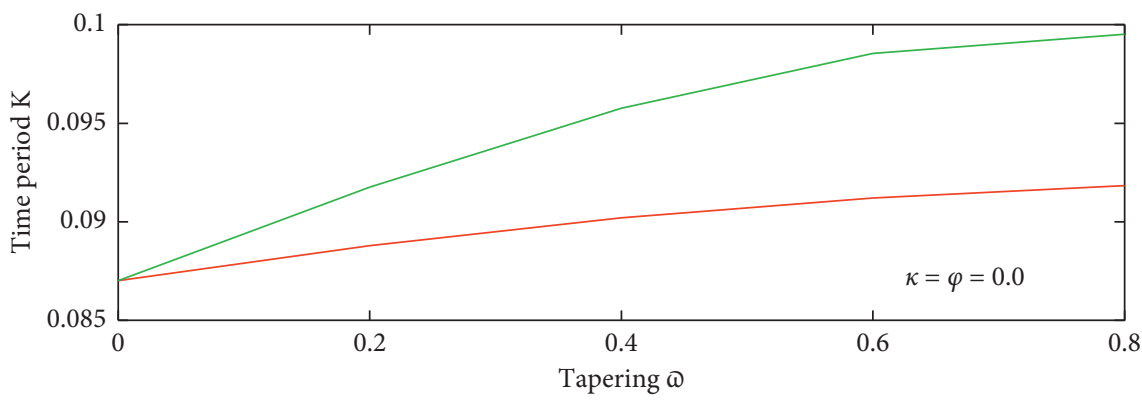

— $\mathrm{K}_{1}$ Present study

$\mathrm{K}_{1}[16]$

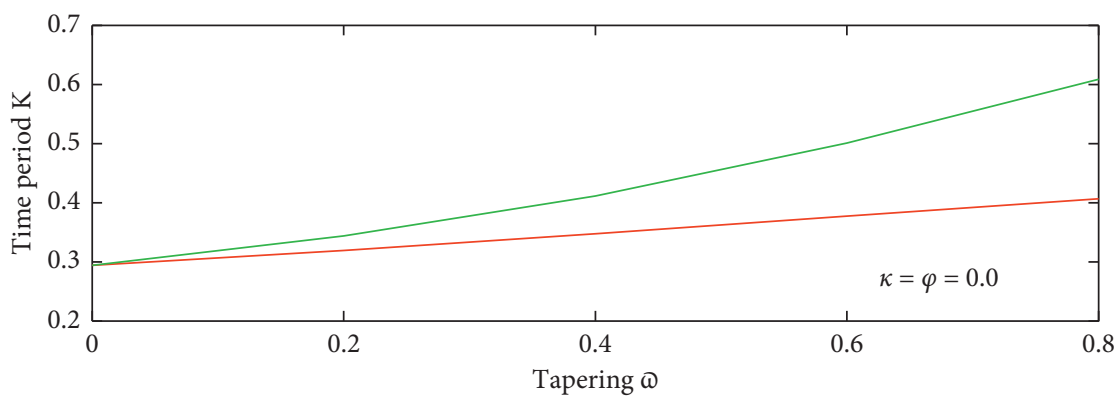

- $\mathrm{K}_{2}$ Present study

- $K_{2}[16]$

FIGURE 9: Comparison of the time period of the clamped circular plate with [16] corresponding to tapering $₫$.
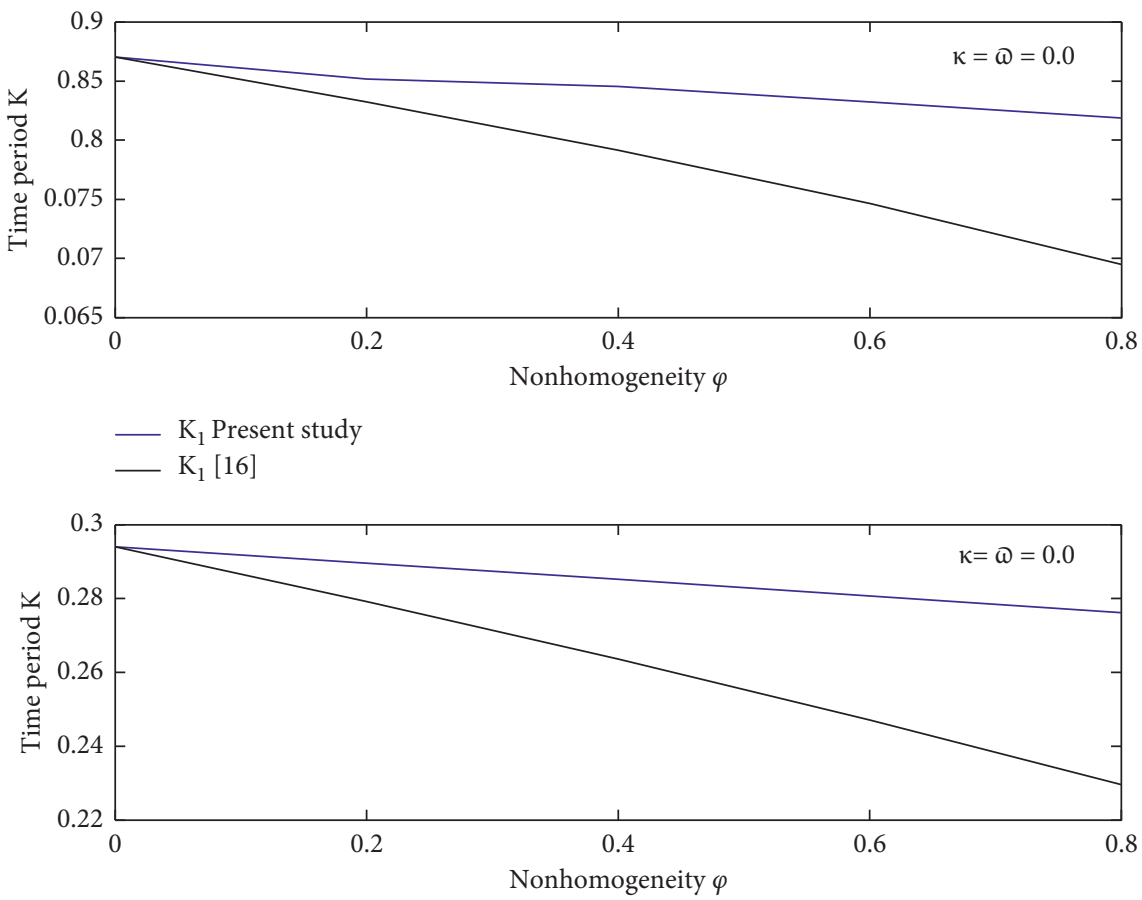

$\mathrm{K}_{2}$ Present study

$-\mathrm{K}_{2}[16]$

Figure 10: Comparison of the time period of the clamped circular plate with [16] corresponding to nonhomogeneity $\varphi$. 


\section{Discussion and Conclusions}

In this section, we will summarize our major findings. The time period for the first three modes of vibration corresponding to the plate parameters, nonhomogeneity, tapering, and thermal gradient was discussed for both clamped and simply supported plates. The study done concluded that, for the case of circular variation in plate parameters, namely, nonhomogeneity and tapering, the time period calculated is smaller in comparison to other variations studied in the literature. Not only this, the change in time period is less rapid as compared to other studies done. For these facts, the mode convergence for circular variation is for a smaller value of $N$ in comparison to other studies where the value of $N$ is higher than our study. This further leads to the conclusion that, for circular variations, the required computational time is less in contrast to other studies.

Based on numerical illustration and comparison, the authors would like to conclude the following facts:

(i) The time period $K$ in case of circular thickness (present study) is less when compared with the time period $K$ in case of linear thickness [16]. The rate of increment in time period $K$ in case of circular thickness (present study) is also less when compared with the rate of increment in time period $K$ in case of linear thickness [16]. The time period $K$ of the present study and that obtained in [16] coincide at $₫=0.0$ (Figure 9).

(ii) The time period $K$ in case of circular density (present study) is higher than the time period $K$ in case of linear density [16], but the rate of decrement in time period $K$ reported in case of circular density (present study) is much less when compared with the rate of decrement in time period $K$ reported in case of linear density [16]. Here also, the time period $K$ of the present study and that obtained in [16] coincide at $\varphi=0.0$ (Figure 10).

(iii) Time period $K$ of clamped and simply supported circular plates increases with increasing value to tapering $₫$ and thermal gradient $\kappa$ (Tables 1 and 2), but the time period $K$ of clamped and simply supported circular plates decreases with the increasing value of nonhomogeneity $\varphi$ (Table 3 ).

(iv) Time period $K$ of the simply supported circular plate is higher than the time period $K$ of the clamped circular plate, but the variation in time period $K$ (rate of increment/decrement) of the clamped circular plate is less when compared with the variation in time period $K$ of the simply supported circular plate (Tables 1-3).

(v) Points (i) and (ii) emphasize that time period $K$ as well as variation in time period $K$ can be controlled by choosing an appropriate variation in plate parameters, while points (iii) and (iv) focus on how the time period $K$ is affected by plate parameters.

\section{Symbols}

$l:$

$r_{1}:$

$\rho$ : $\quad$ Mass density per unit volume of plate

material

v: $\quad$ Poisson's ratio

$V_{s}: \quad$ Strain energy

$T_{s}: \quad$ Kinetic energy

D: $\quad$ Flexural rigidity

E: $\quad$ Young's modulus

$\phi: \quad$ Transverse deflection

$\omega: \quad$ Circular frequency

$\Phi: \quad$ Mode shape function

$\varphi$ : $\quad$ Nonhomogeneity constant

$\kappa: \quad$ Thermal gradient

Ф: Tapering parameter

$\eta$ : $\quad$ Temperature on the plate

$\overline{\Phi_{1}}$ : $\quad$ Deflection function

$\lambda: \quad$ Frequency

$K: \quad$ Time period

$N$ : Number of terms

$K_{i}, i=1,2,3$ : Time period for the first three modes of vibration.

\section{Data Availability}

The research data used to support the findings of this study are currently under embargo, while the research findings are commercialized. Requests for data, 6 months after the publication of this article, will be considered by the corresponding authors.

\section{Conflicts of Interest}

The authors declare that they have no conflicts of interest.

\section{References}

[1] J. S. Tomar, D. C. Gupta, and N. C. Jain, "Vibrations of nonhomogeneous plates of variable thickness," The Journal of the Acoustical Society of America, vol. 72, no. 3, pp. 851-855, 1982.

[2] U. S. Gupta, R. Lal, and S. Sharma, "Vibration analysis of nonhomogeneous circular plate of nonlinear thickness variation by differential quadrature method," Journal of Sound and Vibration, vol. 298, no. 4-5, pp. 892-906, 2006.

[3] J. Yuan and W. Chen, "Exact solutions for axisymmetric flexural free vibrations of inhomogeneous circular Mindlin plates with variable thickness," Applied Mathematics and Mechanics, vol. 38, no. 4, pp. 505-526, 2017.

[4] B. Singh and S. Chakraverty, "Transverse vibration of annular circular and elliptic plates using the characteristic orthogonal polynomials in two dimensions," Journal of Sound and Vibration, vol. 162, no. 3, pp. 537-546, 1993.

[5] S. Bashmal, R. Bhat, and S. Rakheja, "Frequency equations for the in-plane vibration of circular annular disks," Advances in Acoustics and Vibration, vol. 2010, Article ID 501902, 8 pages, 2010. 
[6] B. Uymaz, M. Aydogdu, and S. Filiz, "Vibration analyses of FGM plates with in-plane material inhomogeneity by Ritz method," Composite Structures, vol. 94, no. 4, pp. 1398-1405, 2012.

[7] Y. Wang, R.-Q. Xu, and H.-J. Ding, "Free axisymmetric vibration of FGM circular plates," Applied Mathematics and Mechanics, vol. 30, no. 9, pp. 1077-1082, 2009.

[8] Y. Wang, W. Chen, and X. Li, "Statics of FGM circular plate with magneto-electro-elastic coupling: axisymmetric solutions and their relations with those for corresponding rectangular beam," Applied Mathematics and Mechanics, vol. 36, no. 5, pp. 581-598, 2015.

[9] R. Rana Us, "Damped vibrations of rectangular plate of variable thickness resting on elastic foundation," Applied and Computational Mathematics, vol. 2, no. 3, pp. 1-6, 2013.

[10] K. K. Zur, "Free vibration analysis of elastically supported functionally graded annular plates via quasi-Green's function method," Composites Part B: Engineering, vol. 144, pp. 37-55, 2018.

[11] S. Sharma, R. Lal, and N. Singh, "Effect of non-homogeneity on asymmetric vibrations of non-uniform circular plates," Journal of Vibration and Control, vol. 23, no. 10, pp. 16351644, 2017.

[12] R. Bhardwaj, N. Mani, V. Kumar, and A. Sharma, "Computation of natural vibration of non uniform skew plate with circular variation in density and Poisson's ratio," in Advanced Numerical Simulation in Science and Engineering, pp. 103-113, Amazon Publishing, Seattle, WA, USA, 2020.

[13] A. Sharma, "Vibration of skew plate with circular variation in thickness and Poisson's ratio," Mechanics and Mechanical Engineering, vol. 22, pp. 43-52, 2018.

[14] A. Sharma, "Vibration frequencies of a rectangular plate with linear variation in thickness and circular variation in Poisson's ratio," Journal of Theoretical and Applied Mechanics, vol. 57, no. 3, pp. 605-615, 2019.

[15] A. Sharma, "Vibration of nonhomogeneous square plate with circular variation in density," Engineering Vibration, Communication and Information Processing, vol. 478, pp. 253-263, 2019.

[16] A. K. Gupta and L. Kumar, "Vibration of non-homogeneous visco-elastic circular plate of linearly varying thickness in steady state temperature field," Journal of Theoretical and Applied Mechanics, vol. 48, no. 1, pp. 255-266, 2010.

[17] R. Lal and R. Saini, "Vibration analysis of FGM circular plates under non-linear temperature variation using generalized differential quadrature rule," Applied Acoustics, vol. 158, no. $15,2020$.

[18] A. W. Leissa, Vibration of Plates, NASA SP-160, U.S. Govt. Printing Office, Washington, DC, USA, 1969.

[19] R. Bhardwaj, N. Mani, and A. Sharma, "Time period of transverse vibration of skew plate with parabolic temperature variation," Journal of Vibration and Control, vol. 27, no. 3-4, pp. 323-331, 2021.

[20] G. K. Ramaiah and K. Vijayakumar, "Natural frequencies of polar orthotropic annular plates," Journal of Sound and Vibration, vol. 26, no. 4, pp. 517-531, 1973. 